\title{
Current Status of Degraded Road Bridge Slab Located in Mountainous Area
}

\author{
Yuji Maki ${ }^{1}$, Tuan Minh $\mathrm{Ha}^{2}$, Saiji Fukada ${ }^{2}$, Kazuyuki Torii ${ }^{2}$ and Ryohei Ono ${ }^{1}$ \\ ${ }^{1}$ Division of Environmental Design, Kanazawa University, Kanazawa, Japan \\ ${ }^{2}$ School of Geosciences and Civil Engineering, Kanazawa University, Kanazawa, Japan
}

\begin{abstract}
In the mountainous area of the Hokuriku region, bridges are suffering early deterioration caused by salt damage, alkali-silica reaction (ASR) and frost damage. Under such a regional situation, the load carrying capacity evaluation of these bridges has become an urgent issue. In this study, a statistical method-survival analysis-was carried out by using inspection data for bridges in the Hokuriku region, and the relationship between degradation tendency of bridges and regional characteristics causing salt damage, ASR and frost damage has been studied. In addition, the degradation causes of the reinforced concrete deck of a road bridge which deteriorated early in the mountainous area of the Hokuriku region were investigated. Cylindrical core extraction was carried out to analyze the degradation causes. Polarizing microscopic observation of specimens collected from RC slabs confirmed that cracks developed from the andesite particles and cracks were confirmed to be filled with ASR gels. Therefore, ASR was a cause of the deterioration. Moreover, the degradation in the mechanical properties of concrete due to ASR was also studied and reported. The results show that the reduction in compressive strength and static elastic modulus of both dry and leakage-water parts affected the load bearing capacity of the structure.
\end{abstract}

\section{Introduction}

While the aging of local bridges is progressing, bridge maintenance and management expenses decrease in the fiscal situation of reduction in tax revenue due to the declining birth-rate in Japan. For bridges spanning more than $2 \mathrm{~m}$, the visual inspection is conducted once every five years. Because of the current budget constraint, it is not currently possible to implement repair and reinforcement systematically. In addition, measures are repeatedly carried out without evaluating the residual strength of the bridge after inspection is completed.

In the Hokuriku region, salt damage, alkali-silica reaction (ASR), and frost damage caused severe degradation early in bridges in the mountainous area without warning [1]. Under such a regional situation, how to evaluate the load bearing capacity of these bridges has become an urgent issue. Moreover, the relationship between degradation tendency of bridges and regional characteristics causing salt damage, ASR and frost damage was also a problem that needs to be solved. As a part of the present study, this study performed a statistical analysis-survival analysis [2]-using inspection data for bridges in the Hokuriku region to solve the above problem. To be more specific, the survival time analysis was conducted for reinforced concrete slabs (hereinafter referred to as RC slab) of steel road bridges in the mountainous area constructed for the dam construction in the 1970s.
In addition, as a case study on the destruction process of the RC slab subjected to frost damage in the Hokkaido district which is a snowy region, Mitamura and colleagues [3] reports the effect of freezing and thawing on fatigue life. Besides, Ishikawa et al. [4] reported the damage situation of the RC slab which has been replaced due to fatigue and salt damage caused by the antifreezing agent on the expressway in the Hokuriku district. Using test specimens close to full size, Maeshima et al. [5] analyzed the relationship between ASR deterioration and fatigue durability of RC slab. However, few studies evaluate the current status of RC slabs in the mountainous area of the Hokuriku district where ASR occurred.

Therefore, this study selected the RC deck slab of a steel road bridge from the group of bridges in the mountain area suspected of being deteriorated by ASR (see Figs. 1-2). Then, degradation factors were identified from various analyses by core extraction and visual observation. Then, the current status of the test bridge was evaluated by the change in the mechanical properties, the observation results of ASR gel under the polarized microscope, and the salt content analysis.

\section{Survival time analysis for routes in mountainous areas}

In the Hokuriku district, salt damage, ASR and frost damage are cited as reason leading to early deteriorations 
in concrete structures, but they are closely related to site conditions. In this study, survival time analysis using bridge inspection data was carried out for the purpose of

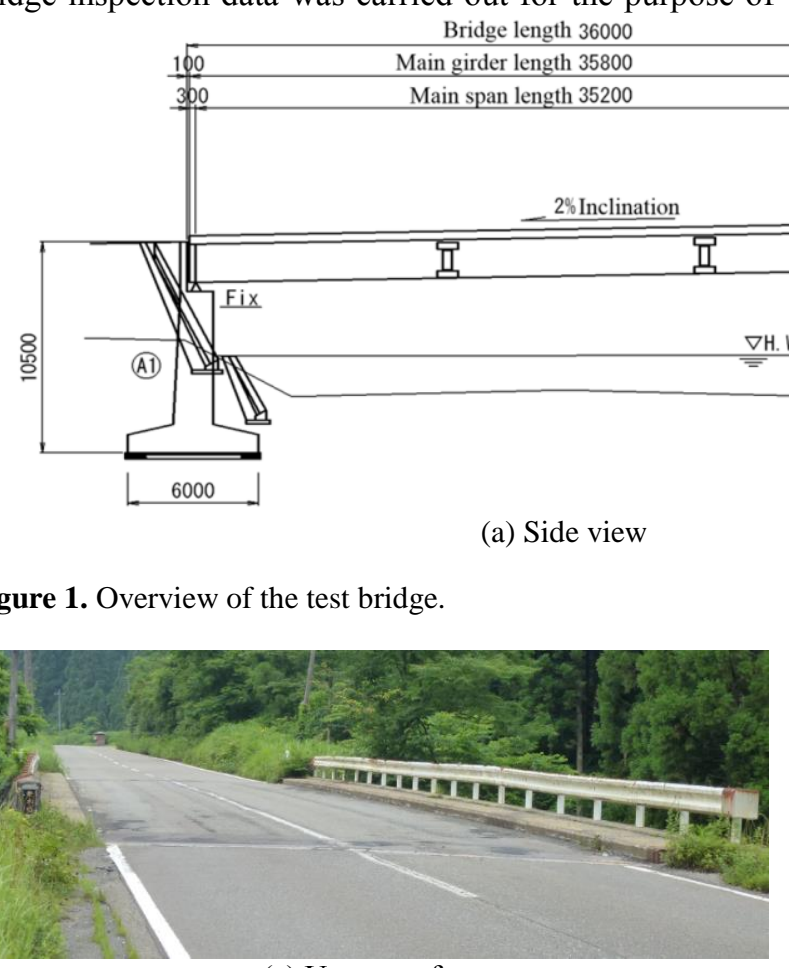

(a) Upper surface

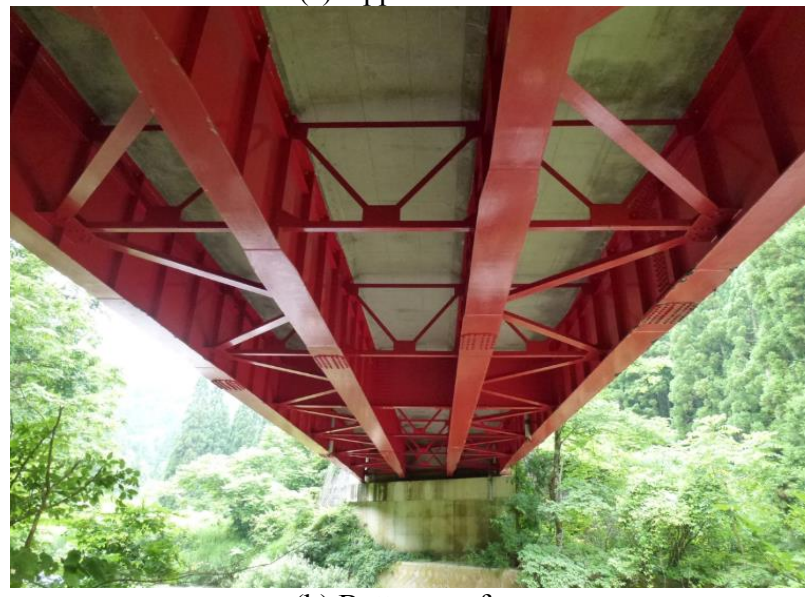

(b) Bottom surface

Figure 2. The test bridge.

\subsection{Outline of survival time analysis}

The survival analysis is a statistical technique focusing on the relationship between time and events from a certain point of time until an event occurs, it is often used in medical and engineering fields.

Although it is common to deal with deaths in the medical field and faults as events in the engineering field, Yamazaki et al. [6] conducted survival time analysis using inspection results in the Tohoku region as an application to the maintenance of bridges and analyzed importance and risk determination for each degradation mechanism.

The survival time $T$ until event occurrence is set as a random variable, the probability density function $f(t)$ is expressed by the following equation: considering the deterioration tendency of the $\mathrm{RC}$ slab in the mountainous routes.

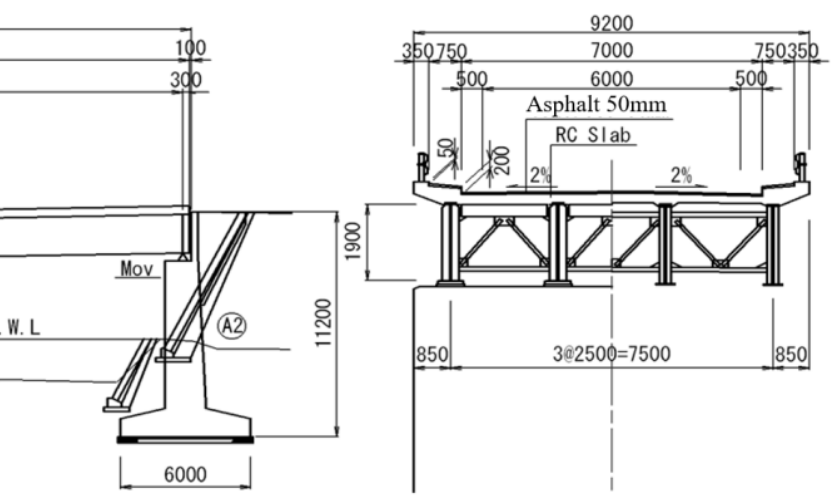

(b) Cross-sectional view

$$
f(t)=\lim _{\Delta t \rightarrow 0} \frac{\operatorname{Pr}(t<T<t+\Delta t)}{\Delta t}
$$

Assuming that the cumulative probability distribution function is $F(t)$, the survival function $S(t)$ at a specific time point $t$ is given by the following equation:

$$
S(t)=\operatorname{Pr}(T>t)=1-\operatorname{Pr}(t \leq T)=1-F(t)
$$

Under the condition that the event has not occurred by the time $t$, the hazard function $h(t)$ indicating the instantaneous mortality rate at which the event occurs at the next moment $t+\Delta t$ is given by the following equation:

$$
h(t)=\lim _{\Delta t \rightarrow 0} \frac{\operatorname{Pr}(t<T<t+\Delta t \mid T \geq t)}{\Delta t}=\frac{f(t)}{S(t)}
$$

Furthermore, the relationship between the cumulative hazard function $H(t)$ and the survival function $S(t)$ and the hazard function $h(t)$ is as follows:

$$
H(t)=\int_{0}^{t} h(t) d t=-\log S(t)
$$

When dealing with the survival time, it is necessary to consider the case where no event is observed before the end of the investigation as "censored". The Kaplan-Meier method is an estimation method considering censoring, and the Kaplan-Meier estimator $S(t)$ is given by the following equation. Here, $d_{i}$ is the number of deaths at time $t_{i}$, and $r_{i}$ is the number of observers that may have an event just before time $t_{i}$.

$$
\hat{S}(t)=\prod_{t_{i}<t}\left(1-\frac{d_{i}}{r_{i}}\right)
$$

\subsection{Survival curve of target route}

\subsubsection{Outline of study}

The bridges over a specific route in a mountainous region in the Hokuriku district were regarded as "applicable group", while other bridges were regarded as "non- 
applicable group". Then, the analysis was performed on both groups to investigate the relationship between the deterioration tendency of the RC slab and the location conditions. Here, the definition of event occurrence was considered as a case where damage requiring repair was found at bridge inspection. According to the inspection record of the district, the bridge soundness was set to 5 grades from grade 5 (mild) to grade 1 (severe). This study considered the healthy degree of grade 3 or less as "damaged", and it was regarded as an event occurrence.

The application of a filter such as classification class and construction year made the comparative examination on both groups possible. The number of steel road bridges having the RC slab in the target area is 276 bridges. Among them, the number of bridges of "applicable" group in the same route constructed for the dam construction in the $1970 \mathrm{~s}$ is 17 bridges. Here, "bridge age" indicates the elapsed year from construction to inspection. Because the RC slabs of the route have not been repaired extensively so far, the restoration of bridge soundness through repair was not taken into consideration, and thereby its impact on the result was considered to be small. For the survival time analysis, the survival package of statistical software $\mathrm{R}$ ver.3.2.5 was employed [7].

\subsubsection{Study results}

Survival time obtained by the Kaplan-Meier method for the "applicable" and "non-applicable" groups is shown in Fig. 3. From this figure, while the survival curve of "nonapplicable" group gradually decreases, "applicable" group shows a sharp decline after 35-45 years. Although there was a difference in the number of samples and the distribution of bridge age in both groups, it became clear that the RC slabs of "applicable" group tended to deteriorate prematurely.

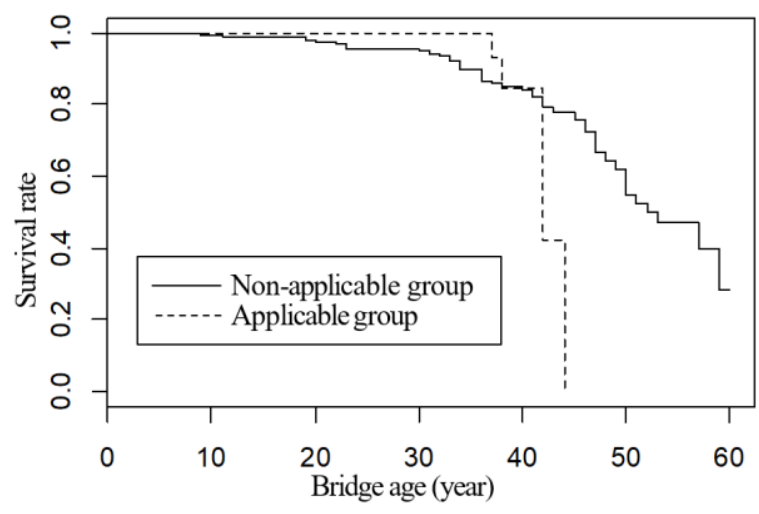

Figure 3. Survival curves of RC slab in target bridge groups.

\section{Description of the test bridge}

As a part of the national highway, the bridge was constructed in the mountainous area (altitude: $559 \mathrm{~m}) 45$ years ago (completed in 1973). From Fig.1, the bridge is a simple composite girder bridge with a bridge length of $36.0 \mathrm{~m}$, and four main girders. Moreover, because the A1 side is lower than the A2 side, the skew angle and the longitudinal gradient are 60 degrees and 2\% respectively. The main girder spacing and the deck thickness are $2.5 \mathrm{~m}$ and $180 \mathrm{~mm}$, respectively. Besides, the volume of traffic (small car + large car) traveling on this bridge and the ratio of large vehicles according to 2015 Census were 1177 vehicles per day and $20 \%$, respectively. Because of the small traffic volume, it was difficult to consider that fatigue was a cause of deterioration in this bridge.

\section{Appearance evaluation}
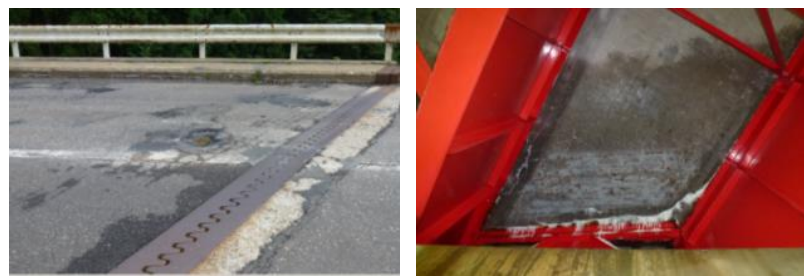

(a) Pavement surface near the joint (b) Bottom surface near the joint

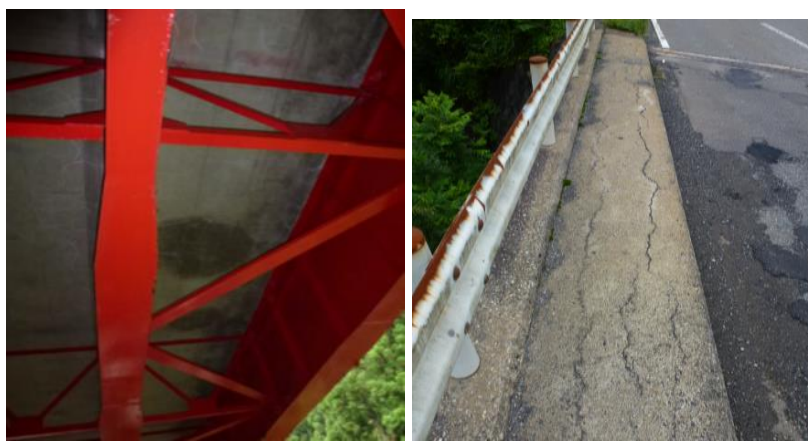

(c) Bottom surface near the span center (d) The wheel guard surface

Figure 4. The test bridge.

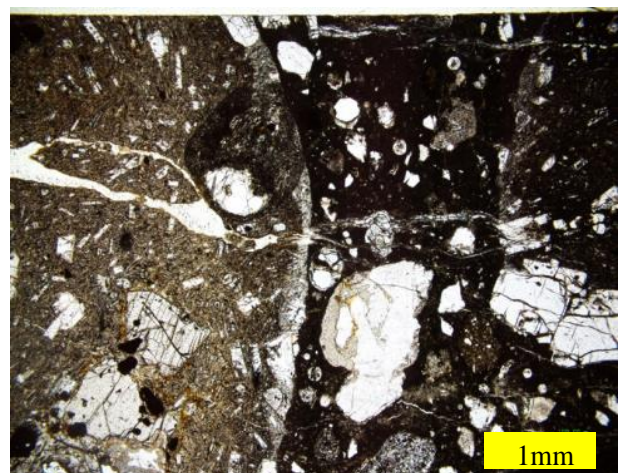

Figure 5. Andesite particles and ASR gel (observation slice 25 $\mathrm{mm} \times 25 \mathrm{~mm}$, thickness $20 \mu \mathrm{m}$ ) under polarizing microscope (normal nicol).

Fig. 4(a) shows the situation of patching of the pavement which seemed to have been repaired several times at the vicinity of the joint. Patches were also confirmed at some places on the pavement. In addition, efflorescence was observed at the bottom of the RC slab and near the joint (see Fig. 4(b)). Signs of water permeation also appeared under the RC slab and near the span center, as shown in Fig. 4(c). Furthermore, the wheel guard section on the bridge surface (Fig. 4(d)) had many horizontal cracks in the longitudinal direction of the bridge. 


\section{Diagnosis ASR-induced deteriorations using drilled cores}

\subsection{Overview of experiment setup}

Cylindrical core samples with a diameter of $55 \mathrm{~mm}$ were collected from the RC slab of the test bridge so as to investigate the mechanical properties of concrete such as the neutralization depth, the compression strength, static modulus of elasticity, and the salinity.

Two sampling locations were selected with and without the efflorescence (dry part, leakage-water part) on the bottom of the slab. The slab thicknesses at the dry part and the leakage-water part (near the joint) are 180 $\mathrm{mm}$ and $190 \mathrm{~mm}$, respectively. The pavement thickness is $50 \mathrm{~mm}$ for both cases.

Fig. 5 shows an observation result under the polarized microscope (normal nicol) of the concrete core collected from the water leakage part. From this figure, cracks propagated from andesite particles to cement paste and the ASR gel was filled in those cracks. Therefore, it was inferred that the main cause of deterioration in the RC slab was ASR.

\subsection{Compressive strength and static elastic modulus}

Compressive strength and static elastic modulus were calculated by performing compression tests using cores collected at two places, i.e., the dry section and the leakage-water section. The results are summarized in Table 1.

From this table, the compressive strength of specimens was reduced by about $30 \%$ in the leakagewater section compared to the dry part. Because the test bridge is a composite bridge and the design strength of concrete was $35 \mathrm{~N} / \mathrm{mm} 2$ for the RC slab, it is inferred the reduction in compressive strength of both dry and leakage-water parts affected the load bearing capacity of the entire structure.

Regarding the static elastic modulus, the results obtained from the leakage-water part decreased to about half of those of the dry part. The previous study pointed out that the elastic modulus was significantly degraded by the ASR-induced deterioration [8], this outcome was also confirmed in results on the RC slab of the test bridge.

Table 1. Compressive strength and coefficient of static elasticity.

\begin{tabular}{|c|c|c|}
\hline & Dry part & Leakage-water part \\
\hline $\begin{array}{c}\text { Static elastic } \\
\text { modulus }\left(\mathrm{kN} / \mathrm{mm}^{2}\right)\end{array}$ & 21.5 & 10.0 \\
\hline $\begin{array}{c}\text { Compressive } \\
\text { strength }\left(\mathrm{N} / \mathrm{mm}^{2}\right)\end{array}$ & 32.3 & 21.2 \\
\hline
\end{tabular}

\subsection{Salt content analysis}

Regarding the salt content analysis of the dry part and the leakage-water part, the sampled cores were sliced in 10 $\mathrm{mm}$ pitch. Then, the total chloride ion content was determined by the potentiometric titration method, and the chloride ion concentration distribution is summarized in Fig. 6. From this figure, the chloride ion concentration in the dry part is almost zero, but in the leakage-water part, it was estimated to be about $1.32 \mathrm{~kg} / \mathrm{m}^{3}$ at the concrete cover of the lower rebar, and the concentration on the lower surface was considerably high. In addition, the measured results did not exceed the limit value of steel corrosion occurrence which could be determined from the proposed formulas $(-3.0(\mathrm{~W} / \mathrm{C})+3.4$; estimated W/C: $55 \%-60 \%$ ) by Standard Specification for Concrete Structures of Japan. In addition, the corrosion of the reinforcing bars was not observed at the place where the core was collected.

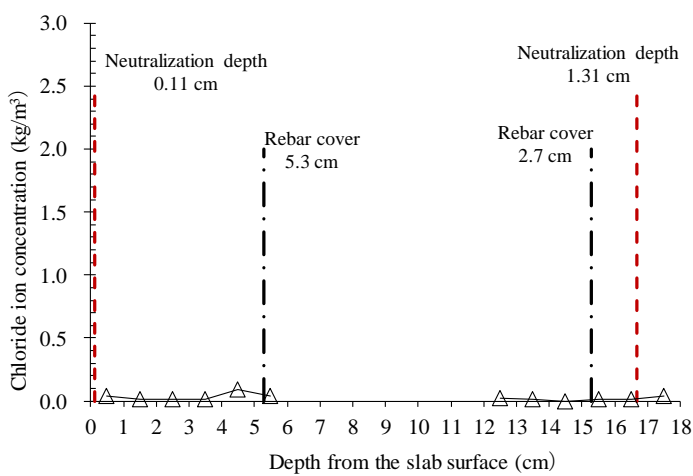

(a) Dry part

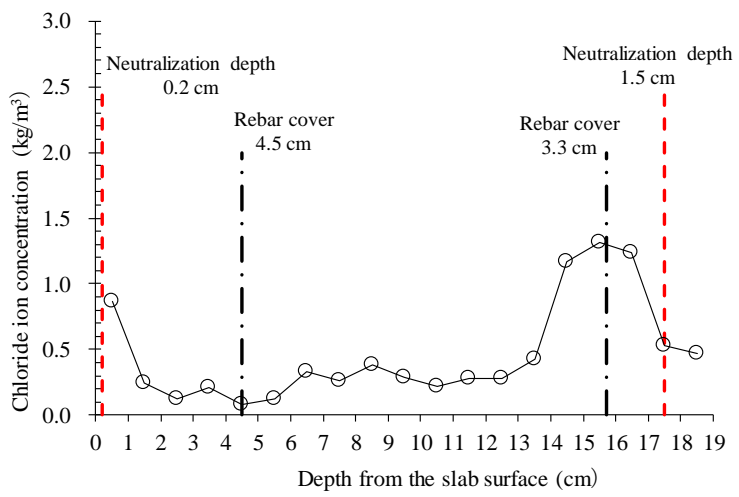

(b) Leakage-water part

Figure 6. Result of salinity analysis.

In addition, the neutralization depth is also shown in same figure. On the lower surface, it was $1.3 \mathrm{~cm}$ in the dry part and $1.5 \mathrm{~cm}$ in the water-leakage part, and neutralization was not progressed as the deck had been over 40 years old. The explanation of this outcome is that rainfall and snowfall frequently occur in the mountains in the Hokuriku district, the humidity continues to be high, and the humid environment slows the progress of neutralization. Also, because the bridge built in the 1970s does not have a waterproof layer, it is presumed that the wet environment causes ASR to progress continuously, resulting in the deterioration of the RC slab.

\section{Conclusions}

This study employed the survival analysis using inspection data for bridges in the Hokuriku district and 
analyzed the relationship between degradation tendency of bridges and regional characteristics causing salt damage, ASR and frost damage. In addition, the degradation causes and current mechanical properties of the reinforced concrete deck of a road bridge which deteriorated early in the mountainous area of the Hokuriku district were investigated by many analyses using cylindrical concrete cores. The primary results obtained from this study are summarized as follows.

Survival time analysis using inspection data was carried out for steel road bridge groups with RC slabs in the mountainous area. It was revealed that the RC slabs of the applicable group were deteriorated earlier than other bridges.

Polarizing microscopic observation of concrete cores collected from RC slabs confirmed that cracks developed from the andesite particles to the cement paste and those cracks were confirmed to be filled with ASR gels. Therefore, it was confirmed that the primary cause of the deterioration in the concrete of the RC slab was presumed to be ASR. In addition, as a result of the investigation on the distribution of chloride ion concentration, corrosion of the steel bar was not confirmed in the leakage-water part as well as the corrosion occurrence limit of the steel material was not exceeded.

As a result of the investigation on the mechanical properties of the cores, the compressive strength decreased by about $30 \%$ in the leakage-water part as compared to the dry part. Regarding the static elastic modulus, the results obtained from the leakage-water part dropped to about half of those from the dry part.

\section{Acknowledgement}

This research was promoted by the Cross-Ministerial Strategic Innovation Promotion Program [Title: Resolution of Early-aged Deterioration Mechanisms in Concrete Bridges and Development of Total Management System Based on Evaluation for Material and Structure Quality Performance, Kanazawa University] from the Japan Science and Technology Agency (JST). The authors wish to thank the concerned parties for their valuable collaboration, sub-consultants, and support.

\section{References}

1. K. Torri, T. Daidai, H. Yamato, T. Hirano, Proceedings of the Japan Concrete Institute, 30, 6 (2008).

2. Y. Maki, Y. Chikata, K. Torri, S. Fukada, Japan Society of Civil Engineers 2017 Annual Meeting, VI524, 2 (2017).

3. H. Mitamura, T. Satou, K. Honda, S. Matsui, J. Struc. Eng., JSCE, 55A, 12 (2009).

4. Y. Ishikawa, Y. Adachi, M. Aoyama, M. Nagai, J. Struc. Eng., JSCE, 57A, 10 (2011).

5. T. Maeshima, Y. Koda, I. Iwaki, H. Naito, R. Kishida, Y. Suzuki, K. Ohta, M. Suzuki, Proceedings of the Japan Society of Civil Engineers E2, 72, 20 (2016).

6. T. Yamazaki, T. Ishida, J. JSCE, Ser. F4 (Construction and Management), 71, 12 (2015).

7. The R Manuals, 2017, https://cran.rproject.org/manuals.html.

8. Y. Kubo, T. Ueda, T. Kuroda, N. Nomura, Proceedings of the Japan Concrete Institute, 28, 6 (2006). 